\title{
HIGHLIGHTS
}

\section{PROSTATE CANCER}

\section{Validation of a prostate cancer risk calculator}

In clinical practice, the decision of whether a man is at risk of prostate cancer and should undergo biopsy is often based on abnormal digital rectal examination (DRE) and elevated serum PSA level. However, the use of predetermined PSA thresholds and DRE findings alone might result in many men undergoing unnecessary, invasive biopsies.

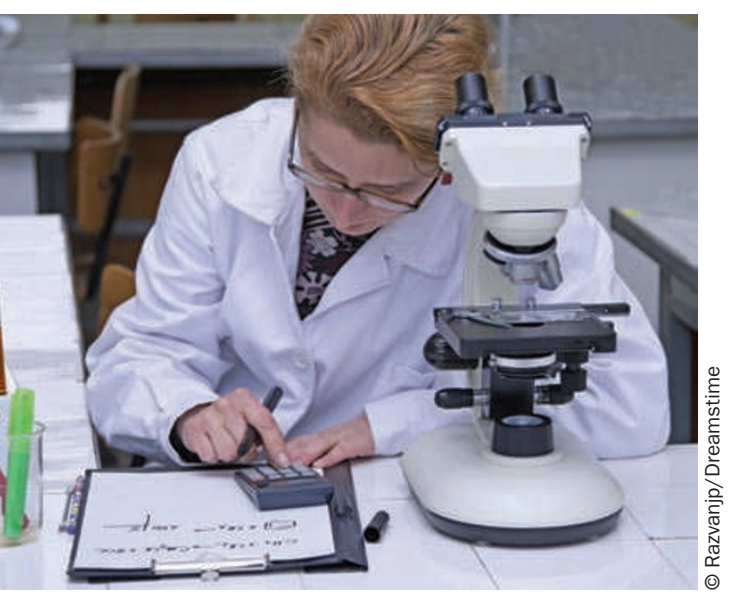

Data from the Prostate Cancer

Prevention Trial (PCPT) have been used to create a 'prostate cancer risk calculator', a nomogram incorporating PSA level, DRE results, previous biopsy findings, age, ethnicity and family history of prostate cancer, to provide risk estimates of biopsydetectable disease. However, the men in the PCPT cohort had low PSA level $(<3 \mathrm{ng} / \mathrm{ml})$, normal DRE findings and were aged 55 years or older, characteristics that are not representative of men routinely referred for prostate biopsy in urology practices. Now, Eyre and colleagues have tested the generalizability of the PCPT calculator in a multicenter cohort of 645 men referred for a first prostate biopsy.

The men in the validation cohort differed significantly from the PCPT population in several characteristics, including younger age, higher PSA level, increased number of abnormal DREs, greater ethnic diversity, and morecommon family history of prostate cancer. In addition, a larger proportion of men had prostate cancer, of a higher grade, in the validation cohort compared with the PCPT cohort.

The PCPT risk calculator was found to be significantly more accurate for predicting prostate cancer than PSA level alone (area under the curve 0.691 versus 0.655), and the risk estimate generated by the PCPT calculator was appropriately calibrated to the actual risk observed in the validation cohort.

These data seem to confirm the generalizability of the PCPT risk calculator and its applicability in routine urology practice. The authors suggest that this tool may prove useful for making individualized decisions regarding the necessity of biopsy.

\section{Nick Warde}

Original article Eyre, S. J. et al. Validation in a multiple
urology practice cohort of the Prostate Cancer Prevention
Trial calculator for predicting prostate cancer detection.
J. Urol. 182, 2653-2658 (2009)

